\title{
ADVANCED PORTABLE SYSTEM FOR STRESSFREE WALKING AND SITTING
}

\section{Saurabh Bhanudas Kandalkar}

\author{
Saurabh Bhanudas Kandalkar, Instrumentation \& Control, Vishwakarma Institute of Technology, Maharashtra, India
}

\begin{abstract}
The basis of this paper is to design and development of portable system for stress free walking and sitting. basically this modular and portable system is a electromechanical system which will help old or aged people and disabled people to walk freely without applying excess stress on their bones, that is this system helps in reduction of stress on legs while walking, also this will help them to walk with more speed than regular. It is also designed for purpose of sitting anywhere with developed structure by just taking support of the wall and without use of chair. This is a modular electromechanical system which is attached externally to legs on outer sides of both feet that means this electromechanical structure is detachable from the body. And the mechanical structure is made in such a way that it will support the back also because at the time of sitting, support to back is required. The design consists of number of simple DC motors of particular specifications, Microcontroller or Microprocessor, mechanical linkages, and other basic mechanical and electronic components. This design can be made in very cheap cost and also portable so that we can take this structure anywhere in car or through any transportation medium and use it, also it utilizes very less space when made at potable condition.
\end{abstract}

Keywords: Electromechanical, Stress Reduction, Portable Design, Modular Design, Cheap Cost, Easily Transportable, Detachable.

\section{INTRODUCTION}

This is an electromechanical structure to enhance the support while walking and sitting routine for old or aged peoples and disabled peoples having walking problems or disability. when the structure is fitted on ones legs it will have connected components which will help that person to walk without putting excess stress[1] on bones or joints therefore the person's movement will be smooth and fast. And also it will help that person to sit at any place just by taking support of wall and without any extra attachment[2]. The basic design of this structure is connecting mechanical linkages beside legs and coupling them with the help of dc motors at certain points like; knee joint and hip joint. that means there will be two such parts because of 2 legs. And then connecting those both parts at hip joint horizontally by another mechanical linkage. and from that linkage one more vertical structure is mounted for back support. the whole system will be consisting of two main supports one is hip supports which will be used for sitting and other is back support which helps in both walking, standing and sitting. This consists of two electromechanical[3] movable joints; one at hip joint and another at knee joint. By this system we can stand easily walk easily and sit easily due to attachment of motors to the system. With the use of qualities of motors like braking while in standing position we can apply brakes to stand straight easily by gaining extra support, and while sitting also applying brakes at particular positions to attain sitting position. This structure is portable that is it can be folded automatically and utilizes very less space, so it can be transported easily this qualities make this structure user friendly and very useful.

\section{CONCEPT AND DESIGN}

This electromechanical structure basically comprises of electrical components like DC motors, controllers, etc. and mechanical components like metal(preferably aluminum[4])linkages, couplers[5], etc. The concept behind this electromechanical system in which the structure is made in such a way that it is modular and detachable. when in use it can be fixed to the body externally, and when not in use detached and packed as it is portable[6]. In this design there are two parts which are connected or attached to legs, bottom part will be attached to ankle and top part will be continued till hip, at hip there will be horizontal linkage joining two parts from both legs. that part which is at hip joint will provide support for both legs and whole body for walking or sitting. and from that part which is at hip there will be small continuation upwards ,towards back and will provide more rigid support to body and will maintain the posture while walking. there is pivot joint at knee and hip position for the free movement of linkage alongside legs.by this type of connection when structure attached it will move along legs. and the structure above hip will not have any pivot joints.as they need not move along, therefore they will provide rigid support to legs and body. At pivot points there is DC motor connected for each joint, that motors will move other part. that means there will be 4 motors for movement of 4 pivot joints. 2 motors will be at horizontal hip bar and other 2 will be at linkages around thighs Motors will be connected to linkages by couplers, at the hip joint motor the coupler used is flexible coupler[7] as their needs horizontal movement[8] of legs and at knee joint there are rigid couplers as no horizontal movement required. activation of 
these motors will move respective parts. Whether the movement of person is forward or it is backward will depend on motion of motor that is clockwise and anticlockwise. by controlling motion of motor we can control the movement of a person. the main aim for this system is to help the person and not to drive person directly, therefore it will provide a bit more force to legs for easy movement. To do such movement first of all the general speed of person is noted by encoder[9] and by controller respective instructions are supplied and motors are driven with bit more force than the general speed of person.

And for sitting a particular posture is been set.at that particular positions the of a persons the pivot points are brought and then the motors are made in brake condition[10]. that will oppose the movement of motors, as the motors will not move the joints will also not move as they are attached to legs. that will help the person to put all is weight on motors as they are in break conditions. that means that they can sit without any chair or external hardware but they just need a support of a wall or any rigid surface on the way. This structure is portable, for making this structure portable that is fold this system we need to control the motors and set them to particular positions to get the required structure. after getting motors to required position then the motors are put in brake condition and we get a folded system just like a suitcase.

\subsection{MECHANICAL DESIGN}
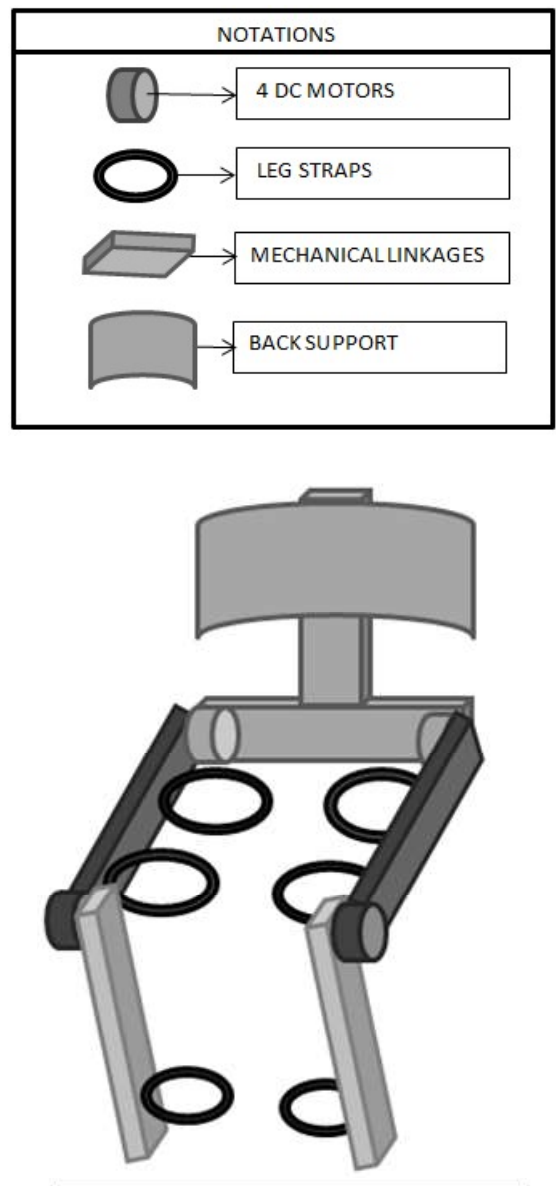

MECHANICALSTRUCTURE OF SYSTEM

\subsection{PORTABILITY}

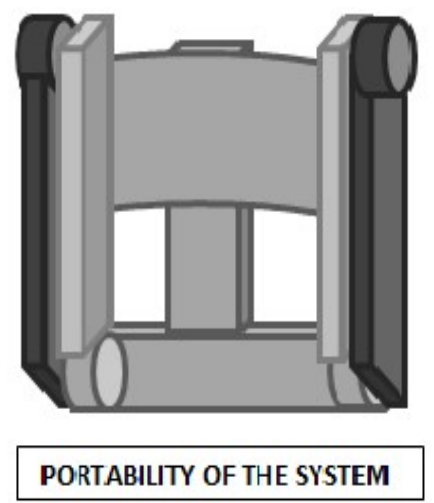

This system is portable that means we can fold this structure in the shape of a suitcase and carry it with the luggage and transport it anywhere for its use. To get it folded the motors connected to it are switched to portable mode availed to the user. By this instruction the motors will reach to their particular position so as to gain the shape of a suitcase, and then they will be put in brake condition until they are put in use by user by its instruction.

\section{PREREQUISITES}

Mechanical components Electronic components

$\begin{array}{ll}\square \text { Aluminum Linkages } & \square \text { DC Motors } \\ \square \text { Nylon Sheet } & \square \text { Motor Drivers } \\ \square \text { Motor Clamps } & \square \text { Microcontroller or Microprocessor } \\ \square \text { Elastic Belts } & \square \text { Encoder } \\ & \square \text { Battery(Lithium-poliymer battery) }\end{array}$

\subsection{DIMENSIONS AND CALCULATIONS}

For Aluminum Linkages

- Total length of average legs is 30 "

- Therefore 4 linkages of 15 " will be required for both legs

- Support linkage for hip will be average 11"

- Linkage for back support required will be $15 "-20$ "

- Back supporting Nylon sheets 10"*5"

For Motor

- Motor is required to move only at $180^{\circ}$ (both knee joint and hip joint movement is max 180').

- By encoder time required for completing that $180^{\circ}$ angle by natural human force is noted down let's say that value as $\mathrm{X}$ (that $\mathrm{X}$ will be actual speed of movement of a person).

- As our main aim is to provide a bit more force than actual to have smooth and stress less movement we add up little more force proportional to actual force to attain those requirements, therefore that RPM will be $(\mathrm{X}+\mathrm{i})$ it will be set by controller and provided to motors for further movements. 
- That $(\mathrm{X}+\mathrm{i})$ will provide required extra force to legs for walking, and further adjustments are provided to a person to increase $\{\mathrm{X}+(\mathrm{i}++)\}$ or decrease $\{\mathrm{X}+(\mathrm{i}--)\}$ the speed than the actual $(\mathrm{X}+\mathrm{i})$.

- Torque required for motor will depend on the weight of the person that is required to move the legs at that particular speed.

- And breaks are applied to all 4 motors at particular angle for sitting.

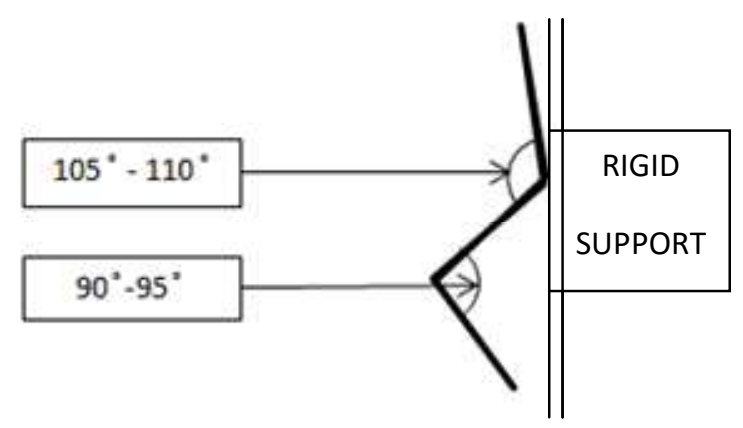

This diagram shows the angles required for motors to put in brake condition for being in sitting position. this angles should be maintained because this will maintain the moment of inertia of the body.

Upper two motors which are at hip joint are braked at $100^{\circ}-110^{\circ}$, and lower two motors which are at knee joint are braked at $90^{\circ}-95^{\circ}$.

\subsection{PROCESS FLOW DIAGRAM}

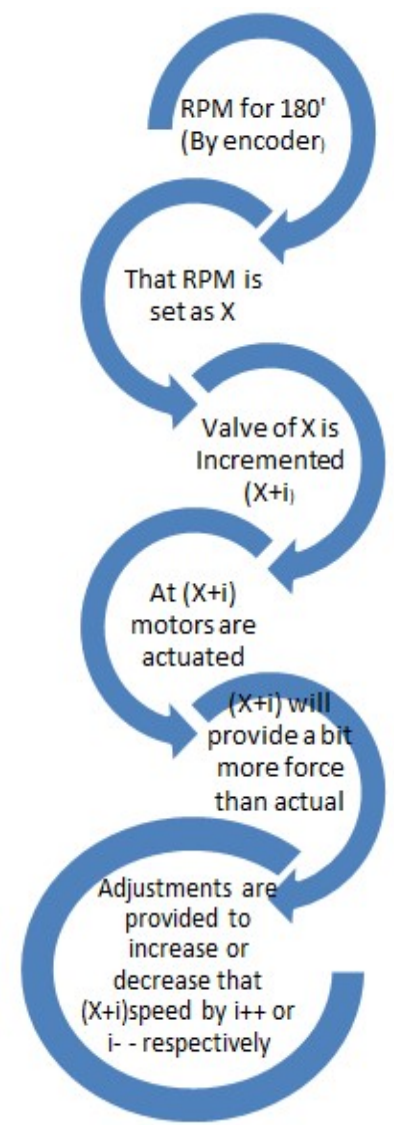

\section{CONCLUSIONS}

The main aim of this system is to provide just more support and force to a person while walking or speed up the walking effortlessly and to help in sitting with use of this structure only. . By this system we get smooth movement of the legs with minimal efforts. Also by provided functionalities of increasing or decreasing speed we can speed up the process if in hurry and speed down the process if exhausted. This system design is modular and portable for its optimized use for the needful peoples. It's portability is one of the important feature because the main structure can be a bit bulky but when it is folded it becomes much smaller and can be carried anywhere easily.

\section{REFERENCES}

[1]. Excess stress is the stress which gets applied on old aged people while walking due to some biological damages [2]. By this design they can sit without use of chair or stick or external attachment

[3]. Electromechanical joints are joints where motors are fixed to get movements like joints

[4]. Aluminum linkages as they are light weighted.

[5].Coupler's are those parts which joins motor and linkages.

[6].Portable is that this structure can be folded and made in smaller shape than previous

[7].Flexible couplers are couplers which provides circular motion while the shaft can move along its other axis.

[8].Horizontal motion is the motion of a leg along its y-axis considering forward motion as $\mathrm{x}$-axis.

[9].Encoder is a devise used to measure RPM of any rotating shaft or body.

[10].Braking condition is a state in which the shaft of the motor tends not to move form its position.

\section{BIOGRAPHY}

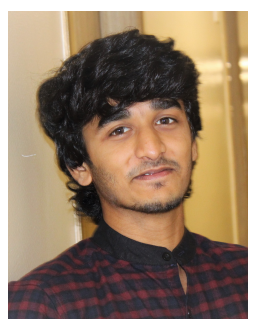

\section{Saurabh Bhanudas Kandalkar}

Studying in final year of Instrumentation and Control engineering department in Vishwakarma Institute of Technology, Pune. I am mechatronics enthusiast and like to design new and different methods for easy and effective solutions for daily life problems. 